\title{
EL LÉXICO VERBAL EN EL BREVIARIUM HISTORIAE ROMANAE DE EUTROPIO ${ }^{1}$
}

\author{
M. a José López de Ayala y Genovés \\ UNED. Madrid
}

\section{RESUMEN}

El Breviarium Historiae Romanae nos ha permitido realizar un estudio del léxico verbal que recoge esta obra. Este trabajo se suma a los ya realizados sobre el Liber memorialis de Ampelio, la Origo gentis Romanorum, y del mismo Eutropio, cuyo objetivo responde a la finalidad del Proyecto «El léxico de la historiografia latina tardía" a través de unas obras de carácter histórico, con fines didácticos y de fácil aprendizaje. En este trabajo no sólo nos remitiremos a su frecuencia, uso y en algunos casos significado, sino también a su presencia en autores precedentes y permanencia en otros, según el espacio temporal abarcado.

El trabajo se enfoca en dos direcciones, la primera relativa al origen y naturaleza del léxico: formas simples, composición y derivación mediante el estudio del uso que de ella se hace en el texto y la segunda, buscando su empleo en los autores latinos anteriores y posteriores al siglo IV, así como su aparición en escritores coetáneos, en prosa y en verso. En cuanto a la primera dirección se ha pretendido ser exhaustivo y en relación con la segunda no se han recogido todos los autores que reflejan los diccionarios sino aquellos que, a nuestro juicio, favorecían el objetivo del trabajo.

1 Este trabajo se realiza en el marco del Proyecto de investigación Léxico de la historiografia latina tardia, financiado or la DGYCIT, 8598-0664. 


\section{INTRODUCCIÓN}

La palabra es analizable ${ }^{2}$ y lo que con esta afirmación se quiere decir es que cualquier variación de sus componentes entraña el cambio de esa palabra por otra o su desaparición. Además tiene un significado gramatical, sintáctico y comunicativo, lo que no exige necesariamente construir una oración, basta usarla sola o combinada gramaticalmente con otras palabras en una situación concreta. Las palabras son diversamente interpretables en sí mismas según su densidad categorial, en donde importa el rasgo (sema), su dependencia funcional o conjunto de condiciones, que regulan la posibilidad de interpretación aislada, y su autonomía contextual o conjunto de condiciones, que regulan las posibilidades de interpretación externa. Por último nos referiremos a la sintaxis como la organización de las palabras en la Lengua, entendida ésta como las posibilidades de combinación de cada palabra con todas las demás, lo que depende de su constitución morfológica o interna.

Todo lo anterior lo hemos recogido porque en una palabra es importante conocer cómo surge, cómo evoluciona y cómo desaparece su significación, ya que tiene un origen individual, pues la crea el individuo que constituye el medio social y de él pasa a la sociedad o al medio social, sin olvidar que no se da aislada sino, como ya hemos dicho, en relación con otras de la misma lengua.

\section{Punto DE PARTIDA}

El trabajo se enmarca dentro de lo que se entiende por la creación de nuevas palabras mediante la adición de afijos derivados a una base léxica, que en este caso, presta especial atención a la prefijación verbal como la anteposición de un afijo a una base léxica y, como consecuencia, a la modificación del significado de la palabra, ya que se trata de un proceso de creación léxica.

El léxico verbal constituye un volumen de gran amplitud y parte de su preverbialidad ha quedado reflejada en el estudio de los prefijos nominales ${ }^{3}$. Centrándonos en el tema que ha sido objeto de estudio, seguimos afirmando que el léxico es el sector de la lengua permanentemente abierto y el más difícil de estructurar. Es necesario distinguir entre su significación fundamental (propio de la Lingüística) y su valor en uso por circunstancias de lugar y tiempo (propio de la Filología). Los mecanismos de renovación del vocabulario latino eran funda-

2 NúÑEz LADEZEvE, L., Teoría y práctica de la construcción del texto, Barcelona, Ariel, 1996, p. 31.

3 LOPEZ de AyAla y Genovés, M." J., «El léxico nominal del Breviarium historiae romanae» de Eutropio, EPOS XV (1999) pp. 13-27. 
mentalmente los mismos que en todas las lenguas: préstamos, composición y derivación. En la estructura de una palabra existen grupos articulatorios que modifican de una manera idéntica o análoga, es decir, los infijos (este sería el caso de -sco), y los que modifican según su posición, a saber, los prefijos o sufijos.

En el caso de los lemas verbales, junto a un gran conjunto de formas simples aparece el procedimiento de la composición que constituye un punto interesante de examen. Dado que el verbo expresa la acción, habría que preguntarse si todos los nombres proceden de una raíz verbal o, por el contrario, tendríamos que examinar en un texto cuál es la frecuencia. Esta estadística nos parece interesante y en el bosquejo que hemos realizado podemos encontrar:

- En primer lugar, lemas procedentes de raíz nominal, así: a) nomen, nominavit en EVTR. 1.2: quos senatores nominavit propter senectutem, EVTR. 7.5: conderent civitates, quas Caesareas nominarent, nominatus, EVTR. 8.14: idem etiam Pius nominatus, y b) dignitas, dignus, dignatus en EVTR.3.5.: In quibus malis nemo tamen Romanorum pacis mentionem habere dignatus est.

- En segundo lugar, lemas procedentes de raíz verbal: amicam: EVTR. 3.3, amicus: EVTR. 1.7, amicitiam: EVTR. 2.9, amor: EVTR. 7.5, amoenitas: EVTR. 6.11; auctor: EVTR. 4.8, auctoritas: EVTR. 9.1; assertor: EVTR. 4.7, assessorem: EVTR. 8.14; arbitrio: EVTR. 3.12; auditorio: EVTR. 9.12; censor: EVTR. 2.4, census: EVTR. 1,7; collega: EVTR. 1.10; conventu: EVTR. 4.4; defensus: EVTR. 8.2, defensor: EVTR.1.10. En este apartado resaltamos los dos lemas siguientes, porque presentan un cambio de significado en latín tardío, así:

- lavacrum: EVTR. 9.4: Romae lavacrum aedificavit, en lenguaje eclesiástico con significado de «Bautismo»,

- pastor: EVTR. 4.7.: Pastor primo fuit, mox latronum dux, en lenguaje eclesiástico referido al «Obispo».

Al hablar de composición, no sólo nos referiremos a la frecuencia de verbos yuxtapuestos, que es importante, sino muy especialmente a los que reunimos bajo el epígrafe de verbos compuestos, siendo su primer elemento un prefijo adverbial que modifica al verbo y expresa una precisón local y temporal del verbo simple, a saber: $a b$ (función extensional), ad (función de aproximación), circum, contra, cum (función sociativa), de (función separativa), dis (función privativa), ex (función intensiva), in (passim, hay que tener en cuenta la acción verbal), inter (función separativa), ob (función obstaculizadora), per (función intensiva), prae (función precedencia, preferencial), pro/prod (función de situación), relred (función de retroceso, repetición, privativa, etc.), sub (función subyacente, supleción), super (función de superposición), trans (función tranversal). Un muestreo de 
ejemplos puede ser el siguiente: accedo; adscendo; circumfero; committere, commovere, concedo, contrago; deferre, demergo, describere; displicere; efficere, exigere, eveho; incedo; intercedo; offero, occido; perdo, permanere, perseverare; praecedo, praesumere; praetermitto; provoco; prodsum; rebello, redigo, removere, reparare, reperire, revocare; subcedo; supervenio; transigo, etc.

Antes de ejemplificar queremos dejar indicado que, de un conjunto aproximado de 800 lemas, nos hemos encontrado, en primer lugar, con un $60 \%$ de formas simples de las que gran parte presentan ejemplos de una única cita; en segundo lugar, el $40 \%$ restante se distribuye de la siguiente manera: 204 registros verbales con formas simples y preverbiales: adnitor, compono, expugno, indico, etc.; 63 registros verbales sin formas simples pero con ejemplos que utilizan sólo un tipo de preverbio: infesto, infido, infinio, etc.; 22 registros verbales sin forma simple y utilizando la forma verbal con varios preverbios: egredior, ingredior, progredior, regredior, transgredior, etc. $\mathrm{y}$, finalmente, un número muy reducido con dos preverbios en la misma forma verbal: incumgnosco; coinquinavit: EVTR. 9.12.: omnibus se sceleribus coinquinavit.

Considerando las formas compuestas podemos hablar de:

a) Lemas temáticos en los que el primer elemento no puede subsistir solo. Ejemplificamos con dos ejemplos a) auspicia EVTR. 2.15: Claudius contra auspicia pugnavit et a Carthaginiensibus victus est $\mathrm{y}$ b) iudices, EVTR. 1.13: quasi proprios iudices et defensores, EVTR. 7.1. En el caso de auspicia, la forma verbal a la que remite el lema no se presenta en el texto en su forma simple, sino con diferentes preverbios que ejemplificamos, a saber: ad-,EVTR. 2.3: ne rectum posset adspicere; cum-, EVTR. 6,16: quo conspecto; por el contrario en el caso de iudices la forma verbal a la que hace referencia el lema, sí se encuentra en su forma simple en el texto: a senatu hostis iudicatus est.

b) Lemas flexivos: cuyo primer elemento puede tener sentido fuera del compuesto: locupletator con el significado de «el que enriquece a otro». Con el significado de título lo encontramos a) aplicado al emperador, como única cita, en EVTR. 10.7: familiarium etiam locupletator (Constantius),y b) referido a Cristo en INSCR. Orell. 816 y GREG.TVR. Thom. 123,16. Otro ejemplo lo tenemos en usurpare: EVTR. 9.11: Quosdam imperium usurpare conatos,

c) Lemas coordinados. Son poco frecuentes y los hemos agrupado de la siguiente manera: a) sustantivo + verbo = causidicis: EVTR. 7.13: fuit convicia a causidicis et philosophis in se dicta leniter tulit; $\mathrm{b}$ ) adjetivo + verbo $=d u$ plicavit: EVTR. 1.6: Hic numerum senatorum duplicavit y también duplicem: EVTR 7.15: duplicem triumphum egit; c) verbo + verbo = patefactas: EVTR. 7.13: quas patefactas ingenti dissimulatione contempsit, y patefecit: EVTR. 3.4.: Alpes, adhuc ea parte invias, sibi patefecit. 


\section{LA LENGUA Y EL ESTILO DE EUTROPIO.}

La lengua y el estilo se conforman a un objetivo didáctico. Si la lengua de Eutropio para algunos estudiosos no es aquella tersa y clásica de la que habla Malcovati ${ }^{4}$, sino que están más en onda con la opinión de Tzschucke ${ }^{5}$ y la de Rebelo Gonçalves ${ }^{6}$ que excluyen toda confrontación con la elegancia de la prosa de la edad áurea. Lo que en ambos casos queda claro es que se trata de una obra, que, aunque realizada por comisión y por motivaciones no literarias, encierra una validez didáctica y pedagógica. Su precisión formular y léxica, su falta de pretensiones retóricas y su aptitud didáctica están en la base del éxito duradero de que ha gozado desde su pronta traducción al griego por Peanio ${ }^{7}$, hasta su uso como manual en la Edad Media.

La función didáctica del Breviarium parece adecuarse muy bien a la estructura de la prosa oficial, como cabe entresacar de las numerosas enumeraciones y de las frecuentes observaciones conclusivas, de resumen o simplemente expositivas del autor Su trabajo es conciso, un estilo simple e intenciones claras, que ofrecen el resultado de un corte personal, totalmente diferente a Aurelio Víctor, con una correcta exposición narrativa, sin omato literario, pero que utiliza un culto lenguaje, donde apenas se advierte el diferente carácter de sus fuentes.

Sobre el plano lexical parece claro que Eutropio no ha usado vocablos diferentes de aquellos que ha podido leer en los documentos de su scrinium ${ }^{8}$. Con este motivo, podemos ejemplificar lo siguiente:

1. Uso frecuente de sustantivos abstractos verbales:

- certamen, EVTR. 2.8: certamine commisso;

- exordium, EVTR. 1.1: a Romulo exordium habet; EVTR. 10.6: qui saevis exordiis dignum exitum nactus est;

- permutatio, EVTR. 2.14: ac permutationem captivorum faceret; status, EVTR. 2.7: nec ante eos ad veterem statum reverti, EVTR. 8.1: respublica ad prosperrimum statum rediit y EVTR. 10.1: Is status erat romanae rei.

4 Malcovati, E., «I Breviari storici del IV secolo» AFLC, 12 (1942) 23-42 y «Le traduzioni greche di Eutropio", RIL 77 (1943-44), 273-304.

5 TzSCHUCKE, C.H., Dissertatio de vita et scriptis Eutropii (con especial atención a la introducción de la misma edición del Breviarium, Lipsiae 1796, p. XXIX, cf. nota del artículo sobre estilística. Incluso se indica también que «In maniera piu completa e sistemática, il vocabolario del nach 1892, nelle seè passato in rasegna da J. Sorn, Der Sprachgebrauch des Historikers Eutropius, Laibach

zioni I A «Gebrauch der Redetheile» e II E «Der Woetschas uestra" de um passo de Eutropio», Huma-

" Rebelo Gonçalves, F. «A expressâo "tranquillitas uestra" nitas 2 (1948), 70.

7 Pavl. DiaC. Ep. ad Adelpergam. of. Droysen, $M G H$. AA. П, $4 \mathrm{sg}$.

\& Spadavecchia, R., I/ ma. Antica Univ. di Padova, 1974-75. 
2. Uso de diferentes voces verbales para expresar la actividad del funcionariado:

- consumere muy frecuente en el Breviarium y afín a nuestro jornalistaburocrático, como vemos en: EVTR. 5.8: insatiabili ira victorum consumpti sunt; EVTR. 8.5: in eo omnis Augusti familia consumpta est; EVTR. 9.9: consumptas paene provincias ... reparaverit.

— distrahere: EVTR. 8.13.: instrumentum regii cultus ... distraxit, con el significado de publice vendere.

3. Uso significativo de sustantivos terminados en -tor y -sor propio de los nomina agentis e indicadores del latín tardío. De estos sustantivos encontramos formas del verbo correspondiente:

- affectator, EVTR. 10.4: Civilibus artibus et liberalibus studiis deditus, affectator iusti amoris y affectans: EVTR.10.1 y 10.4: simul principatum totius orbis affectans;

- coercitor, EVTR. 7.13: diligens tamen coercitor disciplinae militaris y coerceret, EVTR. 1.9: Hinc consules coepere pro uno rege duo hac causa creari, ut, si unus malus esse voluisset, alter eum, habens potestatem similem, coerceret;

- corrector, EVTR. 9.9: Qui quidem Tetricus corrector Lucaniae postea fuit y correctum, EVTR. 4.11: is exercitum ingenti severitate et moderatione correctum, cum nihil in quemquam cruentum faceret, ad disciplinam romanam reduxit;

- pugnator, EVTR. 2.12: XXX cum pugnatoribus cepit y pugnavit, EVTR. 2.8: Curius contra eum pugnavit; expugnavit, EVTR. 3.2: Mediolanum expugnavit; oppugnavit, EVTR.6.3: Lyciae urbes clarissimas oppugnavit. En la forma simple es muy abundante el uso del participio. El uso de pugnator tal como se recoge en EVTR. 2.12. encierra un sentido que se opone a fugitivus, y contrasta con el significado clásico de «combatiente» como se atestigua en Livio, Suetonio, Plinio y con el significado tardío de «campeón» recogido por Celio.Aureliano Ep.2,4;

- receptor, EVTR. 9.9: quasi receptor Orientis Occidentisque y recepisset, EVTR. 6.12: quod regem Tigranem non recepisset y recepit, EVTR. 1.2 (passim): multitudinem finitimorum in civitatem recepit; (passim): recepta, receptae, receptas.

4. Utilización de palabras poéticas o de proveniencia no clásica, casi todas en la tercera parte de la obra (Imperio), tomadas de otros autores posteriores y significativas por el uso vulgar:

- actuarius, EVTR. 9.7: actuario quodam machinante dolum; 
- egit, EVTR. 3.1: Ille gratias Romanis egit y coegit; EVTR. 6.17: postea ad mortem coegit;

- contractis, EVTR. 9.15: per Illyricum Moesiamque contractis copiis;

- exactos, EVTR.1.11: Tertio anno post reges exactos;

- redegit, EVTR. 4.6: ipsum etiam Pseudophilippum in potestatem suam redegit,

- subegit, EVTR.6.3.: Is Ciliciam subegit;

- transegerunt, EVTR. 2,16: quod ambo consules, intra sex dies, quam venerant, transegerunt.

- remeans, EVTR. 10.8: remeansque victor, que aparece sólo una vez con el significado de «regresando victorioso».

5. Puede verse una tendencia al impersonal pasivo, que va implícita en el estilo burocrático y se basa no tanto en la imparcialidad sino en la tendencia a anularse en el servicio del estado, para que los personajes sean vistos más como sujetos que como agentes:

- EVTR. 1.19: a Camillo, qui in vicina civitate exsulabat, Gallis superventum est, gravissimeque victi sunt. En el caso del verbo venio, además de ser muy frecuente en la forma simple, puede encontrase con los preverbios: ad, cum, ex, inter, in, per, prae, sub y super.

- EVTR. 4.27: successum est ei a C. Mario ... et a Cn. Pompeio, maxime tamen a $L$. Cornelio Sulla. Aquí observamos que todas las formas verbales aparecen con preverbio: $a b, c u m, d e, e x$, inter, ob, prae, pro, re, y sub.

6. En algunas ocasiones, las formas preverbiales se usan para completar el sentido de algunos verbos modales:

a) el verbo volo que rige infinitivo: EVTR. 6.5: bellum civile voluit commovere; EVTR. 6.5: Asiam rursus voluit invadere; EVTR. 8.18: Pertinacem se appellari voluit; EVTR. 9.26: et qui severitatem suam aliena invidia vellet explere;

b) el verbo debere con un acentuado valor sentencioso y gnómico como se recoge en: EVTR. 7.21: responderit nullum tristem debere ab imperatore discere.

7. Una de las cuestiones más importantes del Breviarium es la transposición de los tiempos de esse en la forma perifrástica, con la que nuestro autor atestigua cómo incluso en la conciencia lingüística de las personas de un cierto nivel cultural había actuado el «slittamento» temporal ${ }^{9}$ : EVTR. 1,9: com-

" WaGener, C., «Jaharesberichte. Eutropius», Philologus 44 (1885) 324. 
movit tamen bellum urbi Romae rex Tarquinium, qui fuerat expulsus; EVTR. 2,9: pax ...quae cum ipsis propter necessitatem facta fuerat.

Hay que realtar además el uso de un pluscuamperfecto perifrástico con el imperfecto de habere + participio pasado ${ }^{10}$ como en EVTR. 6.16: congregatos habebat; sólo se encuentran formas con el preverbio cum- y en EVTR. 7.20: genituram filiorum ita cognitam habuit; este verbo se presenta con el preverbio re: EVTR 4.6: quae sua recognoscebat.

8. Expresiones que se apartan del uso clásico:

- «subiugare exercitum», frente al uso clásico «sub iugum mittere» en EVTR. 4.8: qua a Numantinis bis romani exercitus fuerant subjugati. Relacionado con esta forma verbal sólo se encuentra, una vez, la forma simple en EVTR. 8.6: cum apud Carnuntum iugi triennio perseverasset.

- remandare, frente al uso clásico "respondere per legatos» en EVTR. 2.7: remandatumque Pyrrho a senatu est; en este caso se encuentra cuatro veces en su forma simple: EVTR. 3.3: ut mandaretur Hannibali.

9. El Breviarium es muy rico en repeticiones de palabras, de locuciones, de estructuras que abundan en las primeras páginas y que debían de haber sido más cuidadas por el autor: EVTR. 3.18.: evocavit ... evocaverunt. Se recogen testimonios con los preverbios: ad-, e-, pro- y re-.

También hay que resaltar las repeticiones cronológicas según el esquema de la fundación de Roma en ejemplos como: EVTR. 2.19 y 3.16: insequento anno; EVTR. 2.10: interiectis aliquot annis y EVTR. 2.14. interiecto anno, que presenta otros preverbios: cum-, in-, ob-y per-, etc.

En la sintaxis " Eutropio presenta repeticiones, que afectan al interior de la oración, sobre todo en el uso de la parataxis y de la yuxtaposición del participio concertado, así en EVTR. 8.4. donde aparecen 11 participios aplicados a la personalidad de Trajano: exhibens ... frequentans ... habens ... sedens ... laedens ... agens ... ditans ... augens ... aedificans ... tribuens ... agens. De este texto y bajo el puento de vista de la composición, nos interesaría resaltar, especialmente, dos: exhibens y aedificans. El primero quedaría recogido en los compuestos de preverbios y el segundo dentro de las formas verbales compuestas, en este caso de sustantivo y verbo, y que aparece en el texto en la forma varias veces reptida de aedificavit: EVTR. 1.6: circum Romae aedificavit; también EVTR. 1.8, 1.9, y 1.14, etc.

11 Cf. VÀÄnÂnen, V., Introducción al Latín Vulgar, trad. Madrid, 1971, p. 211.

"Scivoletto, N., «Dico quod». «Dico quia», GIF 15 (1962) 1-34. 


\section{OBSERVACIONES}

A partir de un muestreo realizado con el $50 \%$ de los verbos prefijados podemos decir que:

1. Los autores clásicos están representados por los historiadores: Nepote, César, Cicerón, Salutio, Tácito, etc. aunque también se encuentran huellas del léxico en autores de poesía como Lucrecio, Virgilio, Ovidio, etc. No todo el léxico mantiene su vigencia en siglos posteriores

2. Es significativo que, frente a un léxico clásico, aparezcan lemas que senalan un léxico tardío, y aunque se encuentra un buen número, aportamos dos ejemplos:

- indulserat, EVTR 10.10: inter cenandum enim epulis indulserat y

- coinquinavit, EVTR. 9.12: omnibus se sceleribus coinquinavit.

3. Las huellas del léxico utilizado por Eutropio se pueden encontrar en autores coetáneos o posteriores a su época, así en Aurelio Victor, Prisciano, Carisio, Celio Aureliano, Agustín, Tertuliano, Paulo Festo, Oribasio, Notas Tironianas, la Collectio Avellana. Citamos algunos ejemplos que también permiten afirmar que, en el uso del léxico, este autor es clásico:

- abstineo: EVTR. 10.8: ut cruore abstineret; en Cipriano se encuentra usado con acusativo de persona;

- administro: EVTR. 7.5: Hoc tamen bellum per Drusum privignum suum administravit; EVTR.6.8: Alter autem Lucullus, qui Macedoniam administrabat, manteniendo el uso clásico, intransitivo y transitivo, frente al uso tardío como intransitivo, según recoge CYPR. Quir. 3.1;

- commendo, EVTR 4.4: Prusias etiam filium suum Nicomedem senatui commendavit, que mantiene el significado clásico «encomendar», frente al figurado que aparece en TERT. Adv.Marc.3.18 y que puede encontrase en otro pasaje de EVTR. 10.9: commendatione patris, con el significado de «verificar, acreditar»;

- converto: EVTR. 5.5: ad Scipionem se convertit; EVTR. 10.8: ad bellum civile conversus. Puede verse que no está usado como verbo deponente, lo que sí aparece en autores tardios;

- decerno, EVTR. 2.13: decrevit senatus ut a maritimis proeliis recederetur, que es sustituido en latín tardío como recoge ORIBAS 562.1 por secerno en sentido figurado;

- indulgeo, EVTR. 10.9: multi exanimatum opinantur nimia cruditate: inter cenandum enim epulis indulserat. Con significado clásico frente al de los 
testimonios de autores tadíos de «perdonar» como lo recoge Prisciano, Casiodoro y otros.

4. Otro lemas, cotejados en los diccionarios y recogidos en Souter ${ }^{12}$, aportan algunas notas a tener en cuenta para seguir afirmando el uso de las construcciones clásicas:

- adicio, con infinitivo más $u t$, lo que no queda reflejado en los ejemplos tanto en el verbo simple como en el compuesto: EVTR. 2.7: iacere vidisset; EVTR. 1.4: adiecto Caelio monte; EVTR. 1.5: Aventinum montem civitati adiecit. Recogemos también otros ejemplos con diferente preverbio en EVTR. 2.11: Regulus ipse in catenas coniectus; EVTR. 7.12: et in Tiberim deiectus; EVTR. 1.14: Octavo decimo anno post eiectos reges; EVTR. 2.8: interiecto anno; EVTR. 10.2: quos etiam bestiis, cum magnificum spectaculum muneris parasset, obiecit; EVTR 7.12: subiecto ad mentum gladio; EVTR. 3.4: e Sicilia exercitum Ariminum traiecit;

- effringo, EVTR. 2.12: neque in aliquo animus his infractus fuit; EVTR. 4.8: quam populus et senatus iussit infringi; EVTR. 6.6: effracto Capuae ludo, con el significado de «abrir con fuerza» que vemos recogido en DON. Aen. 12. 385;

- expugno = oppugno en los ss. III-IV. Hay testimonios en: EVTR. 2.8: Curius contra eum pugnavit; EVTR. 6.3: Lyciae urbes clarissimas oppugnavit; EVTR. 3.8: XXVI expugnavit;

- exsto $=$ sum. No hay ejemplos claros con este sentido en Eutropio, pero nos ofrece lo siguiente: EVTR.7.8: Tam civilis autem circa quosdam amicos exstitit; adstantibus, EVTR. 4.5: cum duobus filits, utroque latere adstantibus; instarent, EVTR.4.7: cum instarent Parthi fugienti; praestarent, EVTR. 4.4: ut liberi essent et dimidium eorum tributorum praestarent;

- incolare $=$ incolere que se recoge en TERT. Ressurr. 26. con el significado de «filtrar, depurar» y que también ha sido recogido por Plinio. Este significado no se encuentra en Eutropio, quien aporta tres ejemplos: EVTR. 6.2: quae Rhodopam provinciam incolebant;

- impleo se encuentra en autores tardíos con el sentido figurado de impono y vemos que se mantiene este uso en clásico acompañado de manum. Esta construcción no se registra en nuestro autor, quien presenta lo siguiente EVTR. 1.15.: promittentes senatui et populo per se omne certamen implendum; EVTR. 8.10: sed Bassiano Antonini nomen a senatu voluit imponi y EVTR. 7.8: filioque suo Britannici nomen imposuit.

\footnotetext{
${ }^{12}$ A Glossary of Later Latin to 600 a. D., compiled by A. SoUTER. Oxford 1949.
} 
5. Nos detenemos en los lemas verbales que aparecen con la cita expresa de Eutropio en los diversos diccionarios, en especial Souter, para recoger lo que aportan sobre el léxico tardío de este autor, a saber:

- edictum referido a la vida pública con el significado de «edicto, orden, una proclamación» aparece en Cicerón y posteriormente en Aurelio Victor, Pomponio, Gelio y en una unica cita en EVTR. 8. 9: qui sub divo Hadriano perpetuum composuit edictum;

- exosus con testimonios en autores anteriores y posteriores al s. IV. Frente al uso clásico «que odia, que detesta» que aparece en Virgilio, Séneca y otros, Souter indica que puede estar usado de forma predicativa como se recoge en Amiano, Rufino, Jerónimo, San Agustín, Macrobio y en la única cita de EVTR. 7.15: Domitianus ... cum ob scelera universis exosus esse coepisset = comenzó a ser odiado por todos;

- exsequiae en su significado propio de «funerales, honras fúnebres» aparece en Plinio. Terencio, Ovidio, Virgilio, Quintiliano, Cicerón y también se recoge en su sentido figurado «restos mortales» en Estacio, Tertuliano, Rufo Festo, Amiano y en: EVTR. 7.13: ut etiam exsequias Neronis, quae humiliter sepultae fuerant, honoraret y EVTR. 9.2: exsequias Romam revexit;

- mando con el significado de «notificar» queda recogido en Tertuliano, Cipriano y Amiano. Presenta la particularidad de que al regir dativo se traduce por «hacer saber» como lo recoge Jerónimo y también EVTR. 5.3: senatuique mandavit bellum se ei [...] illaturum;

- 1 remando: con el significado de «notificar en respuesta» se encuentra en los testimonios de CASS. Hist. Eccl. 10,12. y EVTR. 2.7. Pax dispicluit: remandatumque Pyrrho a senatu est, eum cum Romanis, nisi ex Italia recessisset, pacem habere non posse;

- strata con el significado de «gran ruta» se conserva en testimonios de autores posteriores como AVG. Serm.9,21 y EVTR. 9.9: quod inter Constantinopolim et Heracleam est stratae veteris: locus Coenophrurium appellatur;

- subiugo con el significado de «subyugar, someter» está recogido en CLAUD. Cons. Hon. 6,249; «someter a, obligar a» en DIG. 50,4; LACT. 4,10; «añadir, unir» en TER. MAVR. De Syllab. p. 2395 y, finalmente, con un uso transitivo y significado de «hacer pasar bajo el yugo» en ARN. 4.4. y en la única cita de EVTR. 4.8: A Numantinis bis Romani exercitus fuerunt subiugati.

\section{Conclusión}

Hemos intentado recoger, aunque de manera limitada, el objetivo planteado al comenzar nuestro trabajo, es decir, estudiar preferentemente, a través de 
la ordenación de los vocablos seleccionados por algún criterio más racionalmente didáctico que el basado en el orden alfabético, el uso de la preverbialidad sin dejar de hacer relación a otras formas de composición. Todo ello sin olvidar que la estructura de una lengua está constituida de micro-estructuras, en las que los términos constitutivos mantienen entre ellos relaciones de dependencia, lo que se ha convenido en llamar «campos lingüísticos» ${ }^{13}$ que pueden ser concebidos de diferentes formas: el campo morfológico y el campo semántico. El vocabulario sencillo, notablemente técnico en ocasiones y con muchas locuciones procedentes de la documentación oficial que maneja, no ha dificultado que haya podido realizarse el estudio pretendido, que en un futuro será ampliado. 